57 TIME IN REMISSION AND LOW DISEASE ACTIVITY STATE (LDAS) ARE ASSOCIATED WITH A BETTER QUALITY OF LIFE IN PATIENTS WITH SYSTEMIC LUPUS ERYTHEMATOSUS: DATA FROM A MULTI-ETHNIC, MULTICENTER US COHORT

${ }^{1}$ Manuel F Ugarte-Gil*, ${ }^{2}$ Guillermo J Pons-Estel, ${ }^{3}$ Luis M Vila, ${ }^{4}$ Gerald McGwin Jr, ${ }^{5}$ Graciela S Alarcón. 'Universidad Científica Del Sur; ${ }^{2}$ Grupo Oroño - Centro Regional de Enfermedades Autoinmunes y Reumáticas (GO-CREAR); ${ }^{3}$ Division of Rheumatology. University of Puerto Rico; ${ }^{4}$ School of Public Health. The University of Alabama at Birmingham; ${ }^{5}$ School of Medicine. The University of Alabama at Birmingham

\subsection{6/lupus-2019-|sm.57}

Background Achieving Remission and LDAS are desirable states in lupus patients as they are associated with better longterm outcomes including less damage accrual, lower flare rates and lower disease activity. However, whether achieving Remission and LDAS also account for a better quality of life (QoL) has not been examined. We hypothesized that this will be the case. The aim was to determine whether the proportion of time patients achieve either Remission or LDAS is associated with a better QoL.

Methods SLE patients from a well-established multiethnic, multicenter US cohort were included. Remission and LDAS were defined as follows: Remission, SLAM score $=0$ and prednisone $5 \mathrm{mg} /$ day and no immunosuppressants); LDAS not in remission, SLAM score 3, prednisone $7.5 \mathrm{mg} /$ day, no immunosuppressants; the proportion of time patients were in these two states (combined) was the independent variable. The endpoints were the physical and mental summary measures (PCS and MCS, respectively) and the individual subscales (Physical functioning: FP, Role Physical: RP, Bodily Pain: BP, General Health: GH, Vitality: VT, Social Functioning: SF, Role Emotional: RE and Mental Health: MH) of the Short Form (SF)36 at the last available visit. Linear regression was used to estimate the association between the proportion of follow-up time in remission and LDAS and the SF-36 measures with and without adjustment for the following baseline variables: age, gender, racial/ethnic group, education, poverty, social support, abnormal illness behaviors, fibromyalgia, disease activity, damage and the baseline scores of the corresponding SF-36 summary measures and subscales.

Results Five-hundred and forty-two patients with complete data for the dependent, independent and confounding variables were included. These patients were predominantly women, and either of Caucasian, African American or Hispanic ancestry. Overall, the mean scores for the summary measures of the SF-36 were low (38.9 for the PCS and 43.4 for the MCS); for the individual subscales the scores varied between 40.4 for VT and 65.4 for $\mathrm{MH}$. In the adjusted MV analysis, the percent of time on either Remission and LDAS was associated with better QoL after adjusting for potential confounders (table 1).

Conclusions The percent of time lupus patients stay on Remission or LDAS is associated with a better QoL as measured by summary measures and subscales of the SF-36.

Funding Source(s): None

\section{IDENTIFICATION OF SYSTEMIC LUPUS ERYTHEMATOSUS SUBGROUPS USING ELECTRONIC HEALTH RECORD AND GENETIC DATABASES}

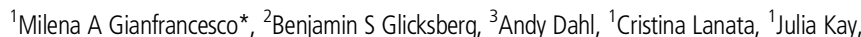
${ }^{1} J o a n n e$ Nititham, ${ }^{1}$ Kimberly Taylor, ${ }^{2}$ Ishan Paranjpe, ${ }^{3}$ Noah Zaitlen, ${ }^{2}$ Marina Sirota, ${ }^{1}$ Lindsey Criswell, ${ }^{4}$ Gabriela Schmajuk, ${ }^{5}$ Jinoos Yazdany. ${ }^{1}$ Division of Rheumatology, Department of Medicine, University of California, San Francisco; ${ }^{2}$ Baker Institute for Computational Health Sciences, University of California, San Francisco; ${ }^{3}$ Department of Neurology, University of California, Los Angeles; ${ }^{4}$ University of California, San Francisco; San Francisco VA Medical Center; ${ }^{5}$ UC San Francisco

\subsection{6/lupus-2019-Ism.58}

Background Systemic lupus erythematosus (SLE) is a multifactorial disease with genetic and environmental risk factors that encompass a wide range of disease severity and heterogeneous manifestations. Long-term outcomes for individual patients are difficult to predict and little is known about why an affected individual might develop a particular SLE phenotype. Identifying nuanced patterns in clinical and molecular data of patients could reveal distinct clusters of disease which could in turn lead to more refined and personalized treatment regimens. Previous studies have used phenotype-mapping approaches to identify subtypes of SLE using genome-wide association studies and gene expression data; however, no studies have integrated both genetic and clinical data from electronic health records (EHR) to identify SLE phenotypes using bioinformatic approaches.

\begin{tabular}{|c|c|c|c|c|c|}
\hline $\begin{array}{l}\text { SF- } \\
36^{*}\end{array}$ & $\begin{array}{c}\text { Number of } \\
\text { patients }\end{array}$ & Estimate & $\begin{array}{l}\text { Standard } \\
\text { error }\end{array}$ & $\begin{array}{c}t \\
\text { value }\end{array}$ & $p$ value \\
\hline MCS & 456 & 5.89 & 1.96 & 3.01 & 0.0027 \\
\hline PCS & 456 & 9.47 & 1.86 & 5.10 & $<0.0001$ \\
\hline FP & 472 & 18.14 & 4.85 & 3.74 & 0.0002 \\
\hline RP & 466 & 31.79 & 6.28 & 5.06 & $<0.0001$ \\
\hline BP & 469 & 19.97 & 4.91 & 4.07 & $<0.0001$ \\
\hline GH & 469 & 23.15 & 3.95 & 5.86 & $<0.0001$ \\
\hline VT & 472 & 13.39 & 4.03 & 3.32 & 0.0010 \\
\hline SF & 468 & 19.03 & 4.78 & 3.98 & $<0.0001$ \\
\hline RE & 463 & 26.28 & 6.37 & 4.13 & $<0.0001$ \\
\hline MH & 472 & 7.51 & 3.81 & 1.97 & 0.0495 \\
\hline
\end{tabular}

*Short Form-36. Mental Component Summary measure; Physical Component Summary measure; Physical functioning; Role Physical; Bodily Pain; General Health; Vitality; Social Functioning; Role Emotional; Mental Health Index. 
Methods We characterized subgroups of patients using sociodemographic and clinical EHR data, and genetic data from previously collected cohorts, for 416 individuals with SLE. Single nucleotide polymorphisms (SNPs) were genotyped on the ImmunoChip. In our analysis, we included 95 variants previously associated with SLE risk. Variables extracted from the EHR included age, sex, race, ethnicity, and disease-associated laboratory results: complement C3 and C4, SSA, SSB, RNP, anti-Smith, and antidsDNA. We first determined subtypes by clustering variables using multi-trait finite mixture of regressions (MFMR), a new clustering method designed for large, multi-trait genome-wide datasets that appropriately accounts for the complex structure of our multi-ethnic dataset. We then used regression analyses to examine whether clinical and genetic variables had differential effects across clusters.

Results Approximately 90\% of patients were female; 52\% were white, 13\% African-American, 13\% Asian, and 22\% other/mixed race. Results demonstrated three distinct clusters (Figure). Cluster $1 \quad(n=165)$ was characterized as predominately white, non-Hispanic/Latino patients with higher age of onset. Cluster $2(n=121)$ had a higher percentage of other/ mixed race individuals with mild disease. Cluster $3 \quad(n=130)$ was categorized by more severe disease, including individuals with a higher percentage of abnormal laboratory values (C3 and C4 levels, +RNP,+anti dsDNA, +SSA, +SSB) and lower age of onset. Eleven SNPs demonstrated significant genotype-cluster interaction with various phenotypes after correction for multiple testing.

Conclusions We identified three distinct subgroups of SLE via unsupervised clustering of sociodemographic and clinical variables derived from EHR and genetic data. Future work will further define these genotype-phenotype clusters and perform validation studies in additional cohorts. Our findings may assist in identifying disease treatments for SLE using a more personalized approach.

Funding Source(s): NIH-NIAMS F32 AR070585 and UCSF PREMIER Core Usage Grant

\section{WHEN STANDARD THERAPY IN PREGNANCY IN WOMEN WITH SYSTEMIC LUPUS ERYTHEMATOSUS AND ANTIPHOSHOLIPID ANTIBODIES IS NOT ENOUGH: THE GLOBAL ANTIPHOSPHOLIPID SYNDROME SCORE}

${ }^{1}$ Massimo Radin* ${ }^{1}$ Irene Cecchi, ${ }^{2}$ Karen Schreiber, ${ }^{1}$ Elena Rubini, 'Dario Roccatello, ${ }^{1}$ Savino Sciascia. ${ }^{1}$ University of Turin; ${ }^{2}$ Department of Thrombosis and Haemophilia, Guy's and St Thomas' Hospital, London, UK

\subsection{6/lupus-2019-Ism.59}

Background Current standard of care (SoC) in pregnancy for patients with Systemic lupus erythematosus (SLE) and/or aPL positivity includes treatment with low dose aspirin $(75100 \mathrm{mg} /$ day) and low molecular heparin or unfractionated heparin. However, up to $30 \%$ of women continue to have pregnancy complications despite SoC(1).

We aimed to assess the validity of the global antiphospholipid syndrome score(GAPSS)(2) in predicting pregnancy morbidity(PM) in patients treated with SoC.

Methods 143 women ever pregnant treated with SoC therapy with SLE and/or aPL positivity were included. Data on cardiovascular risk factors and aPL positivity were retrospectively collected. The individual GAPSS was calculated for each patient by calculating the sum of each risk factor score, as follows: 3 for hyperlipidaemia, 1 for arterial hypertension, 5 for anticardiolipin $\mathrm{IgG} / \mathrm{IgM}, 4$ for anti-2glycoprotein $\mathrm{I} \mathrm{IgG} / \mathrm{IgM}, 3$ for anti-phosphatidylserine/prothrombin antibodies $\operatorname{IgG} / \operatorname{IgM}$ and 4 for lupus anticoagulant. The patients GAPSS was then grouped according to the patients GAPSS into low risk $(<6)$, medium risk (6-11) and high risk (12).

Results The analysis included 143 patients (mean age 30.8 \pm 6.4 ) with SLE $(122 ; 85.3 \%)$ and/or aPL positivity, for a total of 352 pregnancies.

Overall, we observed a live birth rate of $70.5 \%$, with a total of live birth of 248 out of the 352 pregnancies. Fortyfive patients (31\%) experienced at least one event of PM, defined as early or late.

Patients were stratified according to GAPSS values, in order to identify a low risk group (GAPSS $<6, \mathrm{n}=72$ ), a medium

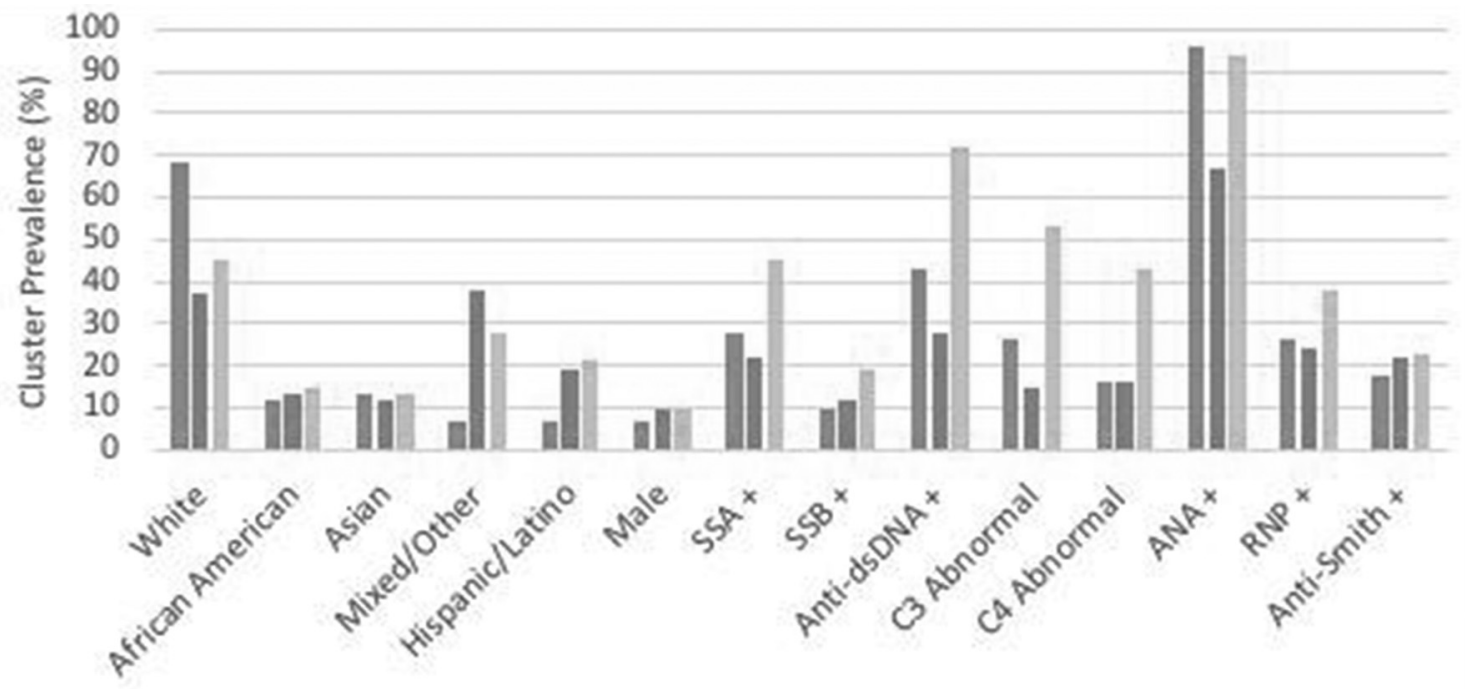

a Cluster 1 a Cluster $2=$ Cluster 3

Abstract 58 Figure 1 Prevalence of demographic and disease characteristics by cluster 